\title{
A novel mutation of FOXC1 in a Chinese family with Axenfeld-Rieger syndrome
}

\author{
XING WU ${ }^{1,2^{*}}$, HAI-NAN XIE ${ }^{2 *}$, TONG WU ${ }^{2}$, WEI LIU ${ }^{2}$, LAN-LAM CHEN $^{2}$, \\ ZHAO-HUI LI ${ }^{1}$, DA-JIANG WANG ${ }^{1}$, YI WANG ${ }^{2}$ and HOU-BIN HUANG ${ }^{1,2}$ \\ ${ }^{1}$ Department of Ophthalmology, Chinese PLA General Hospital, Beijing 100853; ${ }^{2}$ Department of Ophthalmology, \\ Hainan Hospital of Chinese PLA General Hospital, Sanya, Hainan 572000, P.R. China
}

Received November 18, 2018; Accepted June 13, 2019

DOI: $10.3892 /$ etm.2019.7789

\begin{abstract}
Axenfeld-Rieger syndrome (ARS) is a disorder affecting the anterior segment of the eye and causing systemic malformations, and follows an autosomal-dominant inheritance pattern. The aim of the present study was to identify the underlying cause of ARS in a Chinese family. Genomic DNA was extracted from the peripheral blood of the subjects from a family with ARS. The pathogenic variant was identified by targeted next-generation sequencing and confirmed by Sanger sequencing. A novel heterozygous mutation of the forkhead box (FOX)C1 gene (c.1494delG, p.G499Afs*20) was detected in all affected members of the family, while no mutation was identified in the unaffected members or in the 150 normal controls. The affected members exhibited typical ocular and craniofacial anomalies. The results of the present study demonstrated that a novel deletion in exon 1 of the FOXC1 gene caused ARS in this Chinese family.
\end{abstract}

\section{Introduction}

Axenfeld-Rieger syndrome (ARS), occurring in $~ 1 / 200,000$ individuals, is a rare autosomal-dominant genetic disease characterized by anterior segment disorders and systemic abnormalities. The offspring of ARS patients have a 50\% chance of inheriting

Correspondence to: Professor Hou-Bin Huang, Department of Ophthalmology, Hainan Hospital of Chinese PLA General Hospital, 80 JiangLin Road, HaiTang Bay, Sanya, Hainan 572000, P.R. China E-mail: huanghoubin@hotmail.com

\section{*Contributed equally}

Abbreviations: ARS, Axenfeld-Rieger syndrome; FOXC1, forkhead box C1; PITX2, pituitary homeobox 2; FHD, forkhead domain; ARA, Axenfeld-Rieger anomaly; IOP, intraocular pressure; ICE, iridocorneal endothelial syndrome; BCVA, best corrected visual acuity; ASD, anterior segment dysgenesis; SNPs, single nucleotide polymorphisms; InDels; small insertions/deletions

Key words: Axenfeld-Rieger syndrome, forkhead box C1, frameshift mutation, glaucoma, pituitary homeobox 2 the disease. Common anterior segment abnormalities include abnormalities in the anterior chamber angle structure, as well as the cornea and iris. Approximately $50 \%$ of ARS patients eventually progress to glaucoma, which may induce blindness within a small number of years. In addition to the ocular phenotype, systemic developmental abnormalities, including craniofacial hypoplasia (midface hypoplasia, hypertelorism and telecanthus), dental abnormalities (microdontia, oligodontia, adontia and hypodontia), pituitary abnormalities, hearing loss, kidney abnormalities, cardiovascular outflow tract malformation, redundant periumbilical skin, and variable neurological and skeletal anomalies, may also be associated with ARS $(1,2)$.

ARS heterozygotes have less severe and more variable phenotypes than null homozygotes. Affected individuals display overlapping phenotypes, including Axenfeld-Rieger anomaly (ARA) and ARS (3). As a result of the abnormal migration of neural crest cells, ARA encompasses a spectrum of ocular disorders, including iridocorneal adhesions, prominent and anteriorly displaced Schwalbe's line, and atrophic iris. ARS patients present with ocular and systemic malformations.

Two major genes, forkhead box C1 (FOXC1) and pituitary homeobox 2 (PITX2), have important roles in embryonic development and have been demonstrated to cause ARS. Disease-causing copy number changes and point mutations in these two genes have been identified in $40-70 \%$ of ARS patients (4). Mutations in the FOXC1 gene, a member of the FOX family of transcription factors, in ARS patients are more likely to be associated with glaucoma (5). PITX2 is a member of the homeobox gene family and regulates the proliferation and differentiation of various ocular anterior segment tissues and certain non-ocular tissues, including branchial arches and myocardium (6). The mutations in PITX2 are more likely to induce systemic abnormalities than mutations in FOXC1 $(4,7)$.

In the present study, a novel genetic mutation (c.1494delG) was identified in a Chinese family with ARS and the clinical features of the patients were described.

\section{Materials and methods}

Patient recruitment and evaluation. A Chinese Han family from southern China presented with an autosomal-dominant inheritance pattern for ARS (Fig. 1A and 1B) that affected 2 of the 7 living family members. The Chinese family was recruited 
in November 2017. Data on the medical and ophthalmic medical history were collected and ophthalmological examinations were performed. All procedures were performed according to the guidelines of the Declaration of Helsinki for research involving human subjects, and were approved by the ethics committee of the Chinese PLA General Hospital (Beijing, China). Written informed consent, including for DNA extraction, gene analysis and ophthalmological examinations, was obtained from all family members. In addition, 150 normal controls recruited for the present study had provided written informed consent for DNA extraction and gene analysis.

DNA library preparation. Genomic DNA from patients was quantified by agarose gel electrophoresis and Nanodrop ${ }^{\mathrm{TM}}$ 2000 (Thermo Fisher Scientific, Inc.). Libraries were prepared using an Illumina standard protocol (Illumina, Inc.). In brief, $3 \mu \mathrm{g}$ genomic DNA was fragmented by nebulization (E220; Covaris, Inc.). The fragmented DNA was then repaired and an 'A' was ligated to the 3 ' end. Next, Illumina adapters (Illumina, Inc.) were ligated to the fragments, and the sample was size-selected, aiming for a 350-400-base-pair product. The size-selected product was amplified using PCR (each sample was tagged with a unique index during this procedure), and the final product was validated using the Agilent Bioanalyzer 2100 (Agilent Technologies, Inc.).

Targeted gene enrichment and sequencing. The amplified DNA was captured with an ocular disease-associated gene panel with biotinylated oligo-probes using MyGenostics GenCap Enrichment technologies (MyGenostics, Inc.). The probes were designed to tile along 662 ocular disease-associated genes. The capture experiment was performed according to manufacturer's protocol. In brief, $1 \mu \mathrm{g}$ DNA library was mixed with Buffer BL and GenCap gene panel probe (MyGenostics Inc.), and heated at $95^{\circ} \mathrm{C}$ for $7 \mathrm{~min}$ and $65^{\circ} \mathrm{C}$ for 2 min in a PCR machine 2720 (Applied Biosystems; Thermo Fisher Scientific, Inc.). Next, $23 \mu \mathrm{l}$ of the $65^{\circ} \mathrm{C}$ pre-warmed Buffer HY (MyGenostics Inc.) was added to the mix, and the mixture was kept at $65^{\circ} \mathrm{C}$ with the PCR heat lid on for $22 \mathrm{~h}$ for hybridization. MyOne beads (50 $\mu$ l; Thermo Fisher Scientific, Inc.) were washed in $500 \mu 11 \mathrm{X}$ binding buffer 3 times and resuspended in $80 \mu 11 \mathrm{X}$ binding buffer. Subsequently, $64 \mu \mathrm{l}$ $2 \mathrm{X}$ binding buffer was added to the hybrid mix and transferred to the tube with $80 \mu 1$ MyOne beads. The mix was rotated for $1 \mathrm{~h}$ on a rotator. The beads were then washed with WB1 buffer at room temperature for 15 min once and WB3 buffer at $65^{\circ} \mathrm{C}$ for 15 min three times. The bound DNA was then eluted with Buffer Elute. The eluted DNA was finally amplified in 15 cycles using the following program: Initial denaturation at $98^{\circ} \mathrm{C}$ for $30 \mathrm{sec}, 15$ cycles of $98^{\circ} \mathrm{C}$ for $25 \mathrm{sec}, 65^{\circ} \mathrm{C}$ for $30 \mathrm{sec}$ and $72^{\circ} \mathrm{C}$ for $30 \mathrm{sec}$, followed by a hold at $72^{\circ} \mathrm{C}$ for $5 \mathrm{~min}$. The PCR product was purified using SPRI beads (Beckman Coulter, Inc.) according to the manufacturer's protocol. The enrichment libraries were sequenced on an Illumina HiSeq 500 sequencer (Illumina, Inc.) for paired-read $100 \mathrm{bp}$.

The variations were detected by a hereditary eye disease enrichment panel and validated by Sanger sequencing. PCR primer sets were designed using Primer 6.0 (forward, 5'-TACTCT CTGCCTCCGGTCAC-3' and reverse, 5'-TGCTTTGGGGTT CGATTTAG-3'; chr6:1611971-1612380, 410 bp). The products were sequenced using a Bigdye terminator v3.1 cycle sequencing kit (Thermo Fisher Scientific, Inc.) and analyzed on an ABI 3730XL Genetic Analyzer (Thermo Fisher Scientific, Inc.).

Bioinformatics analysis. Following HiSeq 500 sequencing, high-quality reads were retrieved from raw reads by filtering out the low-quality reads and adaptor sequences using the Solexa QA package and the Cutadapt program (https://cutadapt.readthedocs.io/en/stable/), respectively (8). The BWA program (http://bio-bwa.sourceforge.net/) was then used to align the clean-read sequences to the human reference genome hg19 (9). After the PCR duplicates were removed using Picard software (http://broadinstitute.github.io/picard/), the single nucleotide polymorphisms (SNPs) and small insertions/deletions (InDels) were identified using the GATK HaplotypeCaller program (https://software.broadinstitute. org/gatk/). The SNPs and InDels identified were annotated using the ANNOVAR program (http://annovar.openbioinformatics.org/en/latest/) (10). MagicViewer (http://bioinformatics. zj.cn/magicviewer/index.php) was used to view the short-read alignment and validate the candidate SNPs and InDels. Non-synonymous variants were evaluated using 4 algorithms, Ployphen (http://genetics.bwh.harvard.edu/pph2/), SIFT (http://sift.jcvi.org/), PANTHER (www.pantherdb.org) and Pmut (http://mmb.pcb.ub.es/PMut/), as previously described, to determine pathogenicity. The Human Gene Mutation Database was used to support the novelty of the results (http://www.biobase-international.com/product/hgmd).

\section{Results}

Phenotyping

Case III:2 (the proband). The 20-year-old female attended the outpatient clinic of our hospital in November 2017. The proband complained of blurred vision for the past 2 weeks in the right eye. Upon initial examination, the patient's best corrected visual acuity (BCVA) was HM/10 cm OD and 20/100 OS. The intraocular pressure (IOP) measured by Goldmann tonometry reached $50 \mathrm{mmHg}$ OD and $33 \mathrm{mmHg}$ OS. Slit-lamp microscopic examination indicated characteristic corneal posterior embryotoxon in the bilateral eyes. Stromal hypoplasia with corectopia were present in each eye. Iridocorneal adhesion was noted in the right eye. Gonioscopy of the bilateral eyes indicated open angles, peripheral broad-based synechiae, anterior insertion of the iris into the trabecular meshwork and prominent iris processes. By fundoscopy, the cup-to-disk ratio was determined to be 0.9 in the right eye and 0.2 in the left. The corneal diameter of each eye was $12 \mathrm{~mm}$. The corneal endothelial cells in each eye were normal. The central corneal thickness was $589 \mu \mathrm{m}$ in the right eye and $643 \mu \mathrm{m}$ in the left. The axial globe length was $25.4 \mathrm{~mm}$ in the right eye and $20.9 \mathrm{~mm}$ in the left. The eyes of the patient were treated by topical anti-glaucoma medications containing brimonidine tartrate and travoprost. The proband's IOP in the bilateral eyes was well controlled and the BCVA in the right eye improved to 20/200. The visual field defect was severe in the right eye but the visual field in the left eye was normal. Further physical examination revealed midface hypoplasia, hypertelorism, telecanthus and dental malocclusion. The umbilicus was normal. The proband was diagnosed with ARS based on the characteristic ocular and systemic signs (Fig. 2A-H). 
A

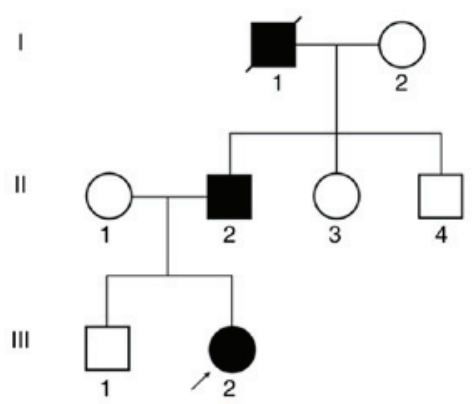

B

$\mathrm{I}: 2$
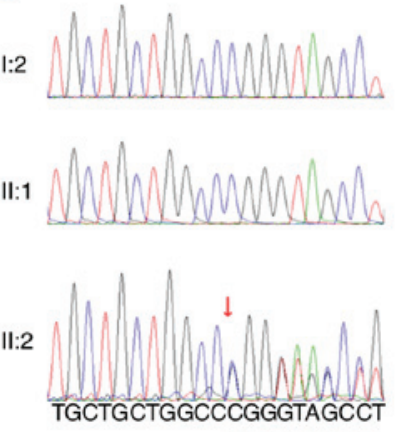

GGGTAGCCTG

$\uparrow$

FOXC1:c. 1494delG
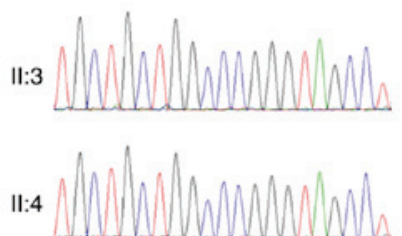

III:1

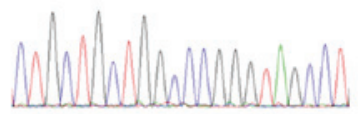

III:2

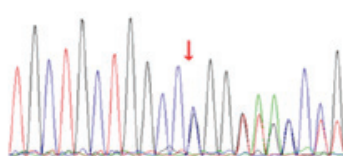

(Based on the principle of complementary base pairing)

Figure 1. Pedigree chart and sequence analysis of a novel mutation (c.1494delG, p.G499 Afs*20) in the FOXC1 gene. (A) In the pedigree chart, the proband (III:2) is indicated with an arrow. The filled symbols represent affected individuals, including the deceased grandfather of the proband. The grandfather was likely to be an ARS patient based on reported medical history and family genetics. (B) Sequences of DNA complementary chains of members of the family affected by ARS. DNA sequencing analysis of the FOXC1 gene was performed. The sequences derived from the affected patients II: 2 and III: 2 indicate a heterozygous mutation c.1494delG (red arrows), resulting in a p.G499Afs*20 frameshift mutation. FOXC1, forkhead box C1.

A

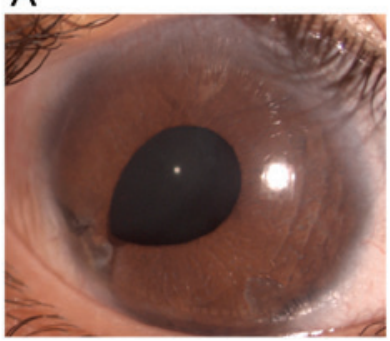

E

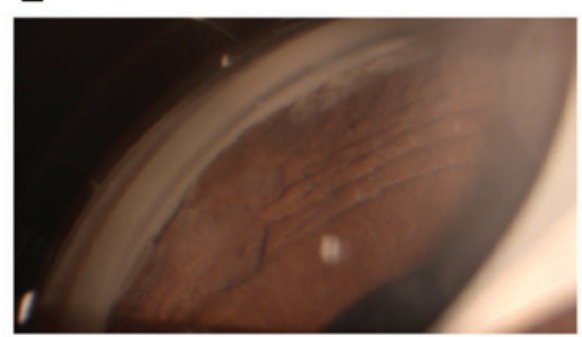

B

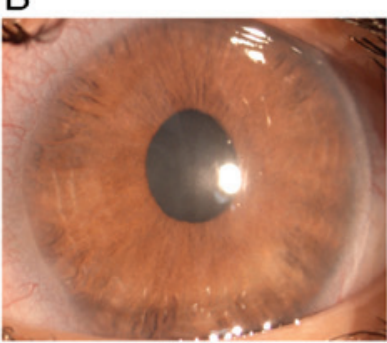

$\mathrm{F}$
C

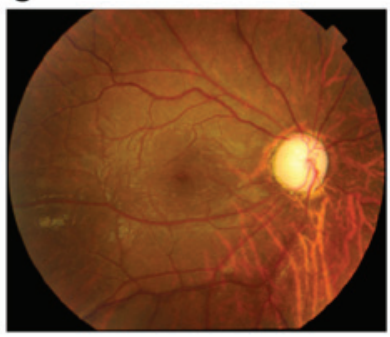

G
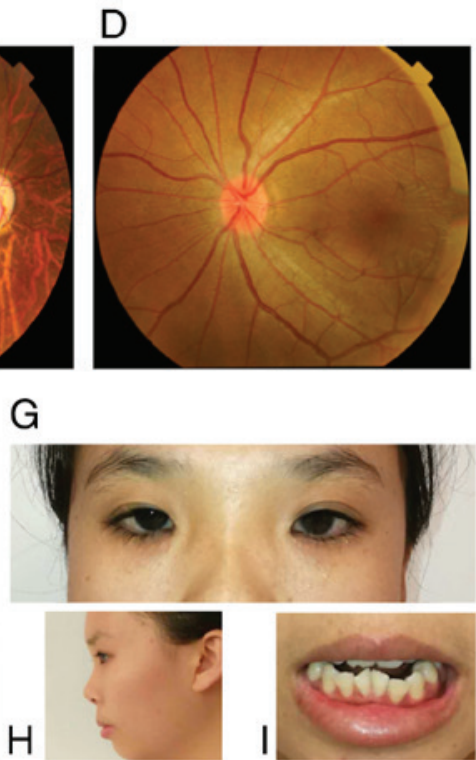

Figure 2. Ocular characteristics and systemic anomalies of patient III:2. (A) Slit lamp images of the right and (B) left eye with posterior embryotoxon and iris hypoplasia. Apparent iridocorneal adhesion and corectopia were noted in the right eye. (C) Fundoscopy indicated that the cup-to-disk ratio was 0.9 in the right eye and (D) 0.2 in the left. (E and F) Gonioscopy revealed open angles with anterior insertion of the iris into the trabecular meshwork, prominent iris processes and broad-based synechiae in the right and (F) left eye. (G) Physical examination revealed hypertelorism, (H) telecanthus midface hypoplasia and (I) dental malocclusion.

Case II:2 (the proband's father). The proband's father was 45 years old. This patient's BCVA was 20/20 in each eye. IOP measured with Goldmann tonometry was $23 \mathrm{mmHg}$ in the right eye and $22 \mathrm{mmHg}$ in the left eye. The iris was atrophic in the bilateral eyes. Gonioscopy indicated open angles in each eye, with prominent iris processes and anterior insertion of the iris into the trabecular meshwork. The patient's visual field test was normal. Further physical examination revealed midface hypoplasia, hypertelorism and telecanthus. The umbilicus was normal. The diagnosis of ARS was made based on the characteristic clinical findings (Fig. 3A-H).

Case I:1 (the proband's grandfather, who had passed away). The proband's grandfather had passed away at the age 85 . The family members of this case reported that he had gone blind due to glaucoma at the age 72 . The grandfather was likely to be a gene variant, which was supported by his medical history and gene analysis of other family members.

Genotyping. The 662 inheritable genetic ocular genes were captured and sequenced by next-generation sequencing using genomic DNA from the proband (III:2). DNA sequencing revealed that two affected members in the family harbored a novel deletion mutation (Fig. 1B). The nucleotide deletion (c.1494delG) within exon 1 in the FOXC1 gene resulted in a frameshift (p.G499Afs*20) mutation, with the destruction of forkhead domain (FHD) structure, which is supposed to result in ARS through haploinsufficiency. 
A

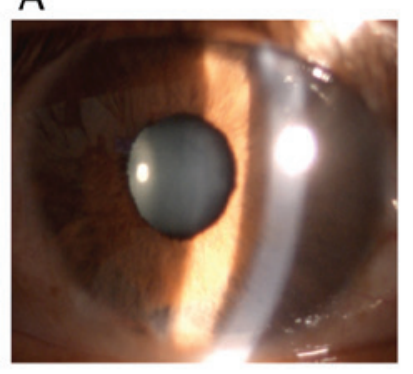

E

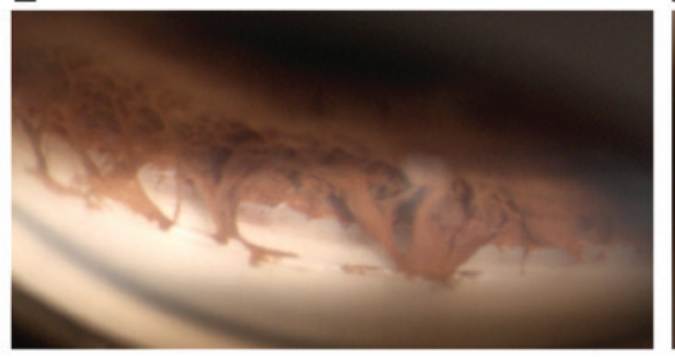

B

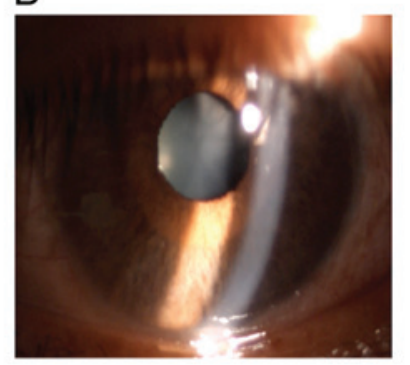

F

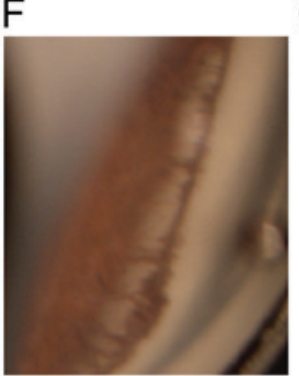

C

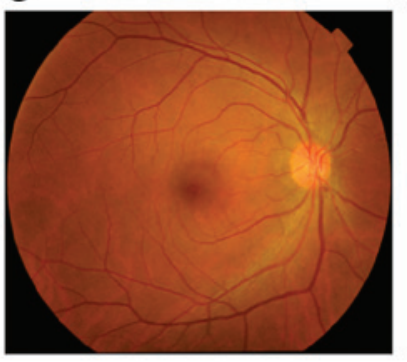

G

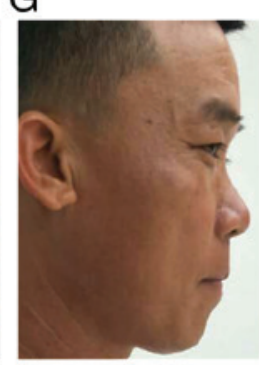

D

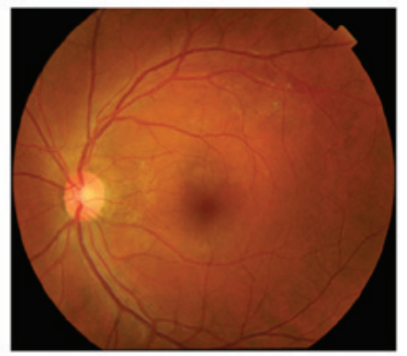

$\mathrm{H}$

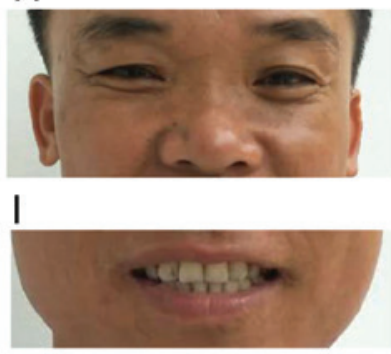

Figure 3. Ocular characteristics and systemic anomalies of patient II:2. (A) Slit lamp images of the right and (B) left eye revealed atrophy of the iris. (C) The fundus of the right and (D) left eye were normal. (E) Gonioscopy indicated open angles in the right and (F) left eye, with prominent iris processes and anterior insertion of the iris into the trabecular meshwork. (G and $\mathrm{H}$ ) Physical examination revealed midface hypoplasia, hypertelorism, telecanthus and flat broad nasal bridge. (I) The dentition was normal.

None of the 5 unaffected individuals (Fig. 1B: I:2, II:1, II:3, II:4 and III:1) had this mutation in the FOXC1 gene. Since case no. I:1 had passed away, gene analysis was not available for this patient. ARS is transmitted by an autosomal-dominant mode of inheritance, so case no. I:1 was likely to be a variant.

The frameshift mutation (p.G499Afs*20) in the C-terminal transactivation domain (aa 466-553) induced a shorter mutant FOXC1 protein (517aa). FOXC1 protein sequence alignment (amino acids 499-517) across species indicates evolutionary conservation at the C-terminal fragment (Fig. 4). The prediction of the protein structure revealed a series of $\alpha$-helices in the C-terminal transactivation domain of the wild-type protein, which was missing in the mutant (Fig. 5). Loss of the series of $\alpha$-helices may have impaired FOXC1 function through altering the transactivation activity, thereby inducing ARS in this family.

\section{Discussion}

ARS is characterized by anomalies in the anterior segment of the eye and systemic signs, and its diagnosis is based on its typical clinical features and genetic defects. There are three types of ARS and each has a different genetic cause. ARS type 1 is caused by mutations in the PITX2 gene. Type 3 is caused by mutations in the FOXC1 gene. The gene that causes Axenfeld-Rieger syndrome type 2 is not known, but it is located on chromosome 13 (11). The two patients in the present study had type 3 ARS.

At times, ARS requires to be distinguished from other anterior segment dysgenesis (ASD) disorders and iridocorneal endothelial syndrome (ICE). ASD disorders encompass a variety of clinical presentations, including ARS, aniridia, Peters anomaly, as well as syndromal associations. ICE is an acquired disorder, is usually unilateral and predominantly affects females in early to middle adulthood. On specular microscopy, corneal endothelial cells of ICE patients were reduced in number and revealed variable degrees of pleomorphism in size and shape, with loss of hexagonal margins. The specular microscopy results of the two patients of the present study were normal.

Most ARS patients presenting with ocular features and with full penetrance are associated with mutations in the FOXC1 and PITX2 genes (11). PR/SET domain 5 and cytochrome P450 family 1 subfamily B member 1 mutations were also reported to be associated with $\operatorname{ARS}(2,12)$. In addition, the association between ARS and PAX6 deletion that was previously reported has recently been demonstrated to be incorrect (13). Two other loci on chromosomes 16q23-24 and $13 q 14$ have been considered to be associated with ARS (11). The most common FOXC1 defects leading to ARS are point mutations (14).

Biochemical analyses of FOXC1 Arg127His demonstrated that although this mutation significantly perturbed the protein function, a patient with this mutation had less severe clinical manifestations than a patient with the biochemically milder mutation FOXC1 Pro79Thr $(15,16)$. Functional analyses of the p.Pro79Thr and p.Pro79Leu mutations indicated that they equivalently inhibited the function of FOXC1, but the impairment of the ARS patient with the FOXC1 Pro79Thr mutation appeared to be more serious than that of the patient with the Pro79Leu mutation $(17,18)$. Therefore, there is no apparent correlation between the functional impairment caused by the missense mutation of FOXC1 and the severity of the phenotype. The phenotype of ARS varies considerably among cases, and even between the two eyes of the same patient $(14,19,20)$. This is consistent with the presentation of the Chinese ARS patients of the present study. The proband presented with glaucoma and severe visual field defect in the right eye but 


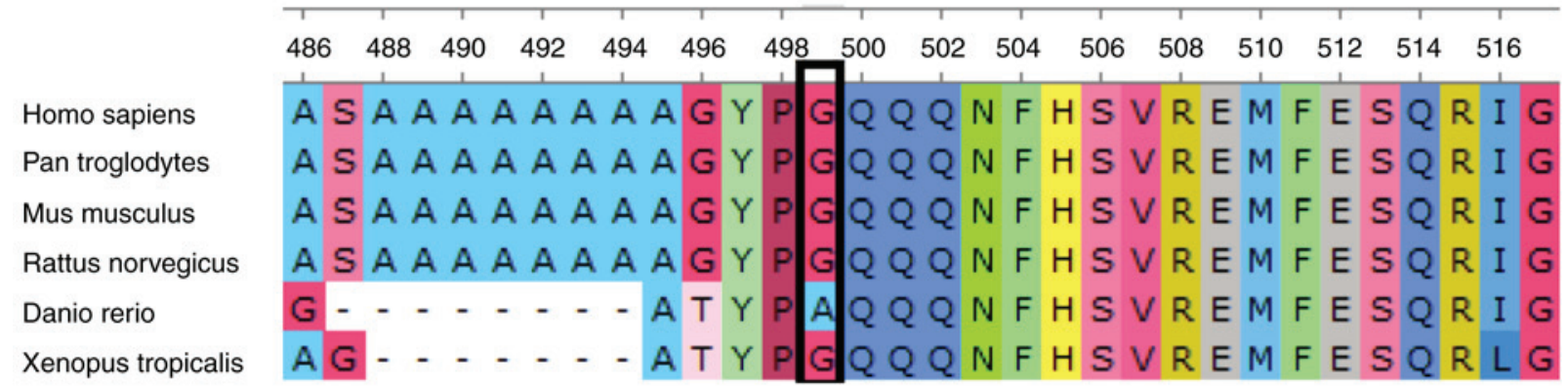

Figure 4. FOXC1 protein sequence alignment. FOXC1 protein sequence alignment (amino acids 499-517) across species, indicating evolutionary conservation at the C-terminal fragment. FOXC1, forkhead box C1.

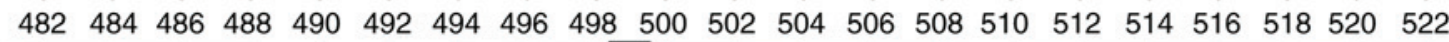

Wild

Mutation

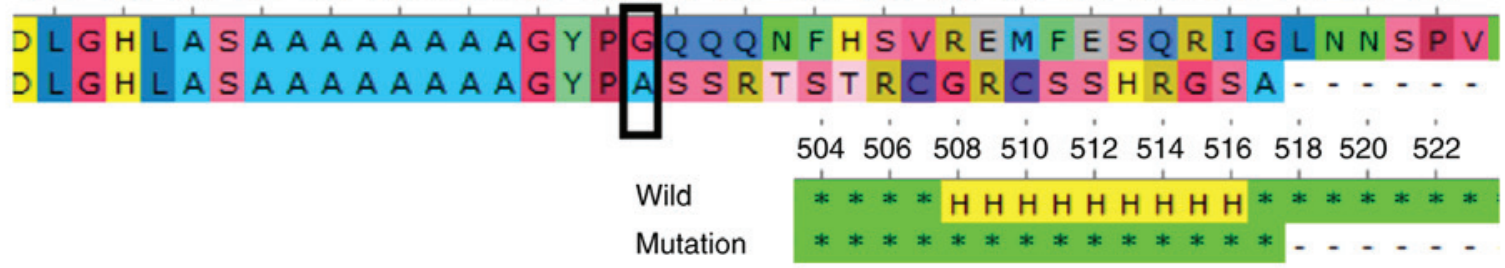

Figure 5. Comparison of amino acids and secondary structure prediction of wild-type and mutant FOXC1. The mutant is a truncated protein of 517 amino acids lacking the series of $\alpha$-helices of the wild-type in the $\mathrm{C}$-terminal transactivation domain. $\mathrm{H}$ indicates $\alpha$-helix. FOXC1, forkhead box $\mathrm{Cl}$; wild, wild-type.

a normal visual field in the left eye. The ocular examinations revealed notable corneal posterior embryotoxon, corectopia and iridocorneal adhesion in the right eye. Gonioscopy of the two eyes indicated peripheral broad-based synechiae and iris strands bridging the iridocorneal angle to the trabecular meshwork. In addition, the proband presented with a flat face, a flat nasal bridge, hypertelorism and telecanthus. The proband was diagnosed with ARS due to genetic defect. The phenotype of the proband's father was considerably milder than that of the proband, and he had normal visual function of the bilateral eyes. Ocular examination of the proband's father indicated slight IOP elevation, atrophy of the iris and anterior insertion of the iris into the trabecular meshwork in each eye. Further physical examination revealed midface hypoplasia, hypertelorism, telecanthus and flat broad nasal bridge. Based on this presentation and the results of the genetic test, the proband's father was diagnosed with ARS.

Genetic defects in PITX2 differ widely and include coding-region frameshift, splice-site, nonsense and missense mutations. FOXC1 and PITX2 interact with each other functionally and physically. FOXC1 is negatively regulated by PITX2. Functional protein dosage alteration, duplication of FOXC1 and deletion of PITX2 are thought to cause similar phenotypes of ARS $(21,22)$. ARS patients with duplication mutations of FOXC1 primarily present with iris hypoplasia and glaucoma, while those with missense mutations have various manifestations with extraocular phenotypes (18).

FOXC1, a 553-amino-acid protein, is characterized by a conserved 110-amino-acid motif known as the FHD. The nuclear localization signals at either end of the FHD support the transfer of FOXC1 to the nucleus and binding to DNA, thereby regulating the downstream target genes. FOXC1 has a phosphorylated inhibitory domain and two transactivation domains located outside the FHD. FOXC1 is highly expressed in the heart, kidney and skeletal muscles, and has a role in tumor development, tissue-specific gene expression and embryogenesis. To the best of our knowledge, 54 different genetic mutations of $\mathrm{FOXC1}$ have been detected in ARS patients, including missense mutations $(n=31)$, InDels/duplications $(n=17)$ and nonsense mutations $(n=6)(9)$. Most missense mutations affect the amino acids within the FHD, which impair the FOXC1 protein function by altering the protein structure, nuclear localization, transactivation activity, DNA-binding ability and protein stability $(16,19)$. The deletions and duplications of FOXC1 induce ARS by affecting the FOXC1 protein level required for the normal development of ocular tissues. Mutations at the C-terminal transactivation domain are less characterized. It has been reported that FOXC1 is a short-lived transcription factor (the half life $t_{1 / 2}<30 \mathrm{~min}$ ) that is degraded through the ubiquitin $26 \mathrm{~S}$ proteasome pathway. Amino acid residues 367-553, which include the C-terminal transactivation domain, are essential for this ubiquitin incorporation and proteolysis, indicating that $\mathrm{FOXC1}$ protein levels and activity are regulated by post-translational modifications (23). The frameshift mutation (p.G499Afs*20), located at the C-terminal transactivation domain, probably influences $\mathrm{FOXC1}$ protein ubiquitination. It may therefore be suggested that it is the underlying cause of ARS in the Chinese family of the present study.

ARS was diagnosed in this family based on ocular findings and craniofacial features. An increased IOP was detected in the two ARS patients. Glaucoma was diagnosed in the female patient (case III.2). The grandfather of this proband (case I.1) had gone blind due to glaucoma; he was likely to be a gene variant and passed on the mutation to his son 
(case II.2). Characteristic ocular features were observed in the proband, including iris insertion into the trabecular meshwork, corectopia, iridocorneal adhesions, posterior embrytoxon and atrophic iris. Although the severity of glaucoma is linked to the level of iris insertion into the trabecular meshwork, physical occlusion of the angle structure is not a prerequisite for glaucoma in ARS patients (24). The female patient of the present study responded well to topical anti-glaucoma combination therapy. Trabeculotomy-trabeculectomy surgery has been proved to be a safe and effective procedure for ARA patients with glaucoma (25). It has been reported that a relatively low proportion of patients with glaucoma and either FOXC1 or PITX2 mutations (18\%) respond to medication or surgery. In comparison with other mutation types, FOXC1 duplication mutants have been associated with a relatively poor prognosis regarding glaucoma development (16).

In the present study, a novel mutation was identified in a Chinese family with ARS and the clinical presentation and eye examination results of the affected patients, including craniofacial and ocular abnormalities, were described. A heterozygous deletion of the FOXC1 gene (c.1494delG) was detected. This 1-bp deletion caused a frameshift mutation, p.G499Afs*20, in the C-terminal transactivation domain, disrupted the secondary structure of the $\mathrm{FOXC1}$ protein and influenced protein ubiquitination. The present results further supported the critical control of $\mathrm{FOXC1}$ in ocular development and broadened the spectrum of FOXC1 mutations in Chinese patients with ARS.

\section{Acknowledgements}

Not applicable.

\section{Funding}

This study was supported by grants from the Special Research and Trial Production Project in Sanya (grant no. 2017KS03), the Science and Technology Achievement Transformation Project in Sanya (grant no. 2017CZ13), the Key Research Plan of Hainan Province (grant no. ZDYF2018139), the Innovation project for Medical and Health Science and Technology in Sanya (grant no. 2014YW35) and the Natural Science Foundation of Beijing Municipality (grant no. 7162180).

\section{Availability of data and materials}

The data supporting the conclusions of the present study are contained within the manuscript. All raw data generated/used during the present study are available from the corresponding author on reasonable request.

\section{Authors' contributions}

XW and NHX performed the analysis of data and drafted the manuscript. HZL and JDW participated in the analysis and interpretation of data. TW, WL, LLC and YW performed the acquisition of data. BHH made substantial contributions to the conception and design of the study and revision of the manuscript. All authors approved the final manuscript as submitted and agree to be accountable for all aspects of the work.

\section{Ethics approval and consent to participate}

The study was approved by the Ethics Committee of the Chinese PLA General Hospital (Beijing, China) and the experiments followed the tenets of the Declaration of Helsinki.

\section{Patient consent for publication}

Written informed consent was obtained from all patients to publish their cases and the photos in this study.

\section{Competing interests}

The authors declare that they have no competing interests.

\section{References}

1. Hjalt TA and Semina EV: Current molecular understanding of Axenfeld-Rieger syndrome. Expert Rev Mol Med 7: 1-17, 2005.

2. Millá E, Mañé B, Duch S, Hernan I, Borràs E, Planas E, Dias Mde S, Carballo M, Gamundi MJ and Spanish Multicenter Glaucoma Group-Estudio Multicéntrico Español de Investigación Genética del Glaucoma, EMEIGG: Survey of familial glaucoma shows a high incidence of cytochrome $\mathrm{P} 450$, family 1 , subfamily $\mathrm{B}$, polypeptide 1 (CYP1B1) mutations in non-consanguineous congenital forms in a Spanish population. Mol Vis 19: 1707-1722, 2013.

3. Espinoza HM, Cox CJ, Semina EV and Amendt BA: Amolecular basis for differential developmental anomalies in Axenfeld-Rieger syndrome. Hum Mol Genet 11: 743-753, 2002.

4. Reis LM, Tyler RC, Volkmann Kloss BA, Schilter KF, Levin AV, Lowry RB, Zwijnenburg PJ, Stroh E, Broeckel U, Murray JC and Semina EV: PITX2 and FOXC1 spectrum of mutations in ocular syndromes. Eur J Hum Genet 20: 1224-1233, 2012.

5. Challa P: Glaucoma genetics. Int Ophthalmol Clin 48: 73-94, 2008.

6. Gage PJ and Camper SA: Pituitary homeobox 2, a novel member of the bicoid-related family of homeobox genes, is a potential regulator of anterior structure formation. Hum Mol Genet 6: 457-464, 1997.

7. Tümer $Z$ and Bach-Holm D: Axenfeld-Rieger syndrome and spectrum of PITX2 and FOXC1 mutations. Eur J Hum Genet 17: 1527-1539, 2009.

8. Cox MP, Peterson DA and Biggs PJ: SolexaQA: At-a-glance quality assessment of Illumina second-generation sequencing date. BMC Bioinformatics 11: 485, 2010.

9. $\mathrm{Li} \mathrm{H}$ and Durbin R: Fast and accurate short read alignment with Burrows-Wheeler transform. Bioinformatics 25: 1754-1760, 2009.

10. Wang K, Li M and Hakonarson H: ANNOVAR: Functional annotation of genetic variants from high-throughput sequencing data. Nucleic Acids Res 38: e164, 2010.

11. Seifi $M$ and Walter MA: Axenfeld-Rieger syndrome. Clin Genet 93: 1123-1130, 2018.

12. Micheal S, Siddiqui SN, Zafar SN, Venselaar H, Qamar R, Khan MI and den Hollander AI: Whole exome sequencing identifies a heterozygous missense variant in the PRDM5 gene in a family with Axenfeld-Rieger syndrome. Neurogenetics 17: 17-23, 2016.

13. Riise R, D'haene B, De Baere E, Grønskov $K$ and Brøndum-Nielsen K: Rieger syndrome is not associated with PAX6 deletion: A correction to Acta. Ophthalmol Scand 2001:79: 201-203. Acta Ophthalmol 87: 923, 2009.

14. Kim GN, Ki CS, Seo SW, Yoo JM, Han YS, Chung IY, Park JM and Kim SJ: A novel forkhead box C1 gene mutation in a Korean family with Axenfeld-Rieger syndrome. Mol Vis 19: 935-943, 2013.

15. Kawase C, Kawase K, Taniguchi T, Sugiyama K, Yamamoto T, Kitazawa Y, Alward KL, Stone EM, Nishimura DY and Sheffield VC: Screening for mutations of Axenfeld-Rieger syndrome caused by FOXC1 gene in Japanese patients. J Glaucoma 10: 477-482, 2001.

16. Saleem RA, Banerjee-Basu S, Berry FB, Baxevanis AD and Walter MA: Structural and functional analyses of disease-causing missense mutations in the forkhead domain of FOXC1. Hum Mol Genet 12: 2993-3005, 2003. 
17. Suzuki T, Takahashi K, Kuwahara S, Wada Y, Abe T and Tamai M: A novel (Pro79Thr) mutation in the FKHL7 gene in a Japanese family with Axenfeld-Rieger syndrome. Am J Ophthalmol 132: 572-575, 2001.

18. Strungaru MH, Dinu I and Walter MA: Genotype-phenotype correlations in Axenfeld-Rieger malformation and glaucoma patients with FOXC1 and PITX2 mutations. Invest Ophthalmol Vis Sci 48: 228-237, 2007.

19. Seifi M, Footz T, Taylor SA and Walter MA: Comparison of bioinformatics prediction, molecular modeling, and functional analyses of FOXC1 mutations in patients with Axenfeld-Rieger syndrome. Hum Mutat 38: 169-179, 2017.

20. Honkanen RA, Nishimura DY, Swiderski RE, Bennett SR, Hong S, Kwon YH, Stone EM, Sheffield VC and Alward WL: A family with Axenfeld-Rieger syndrome and peters anomaly caused by a point mutation (Phe112Ser) in the FOXC1 gene. Am J Ophthalmol 135: 368-75, 2003.

21. Berry FB, Lines MA, Oas JM, Footz T, Underhill DA, Gage PJ and Walter MA: Functional interactions between FOXC1 and PITX2 underlie the sensitivity to FOXC1 gene dose in Axenfeld-Rieger syndrome and anterior segment dysgenesis. Hum Mol Genet 15: 905-919, 2006
22. Seifi M, Footz T, Taylor SA, Elhady GM, Abdalla EM and Walter MA: Novel PITX2 gene mutations in patients with Axenfeld-Rieger syndrome. Acta Ophthalmol 94: e571-e579, 2016.

23. Berry FB, Mirzayans F and Walter MA: Regulation of FOXC1 stability and transcriptional activity by an epidermal growth factor-activated mitogen-activated protein kinase signaling cascade. J Biol Chem 281: 10098-10104, 2006.

24. Schachtschabel DO, Binninger EA and Rohen JW: In vitro cultures of trabecular meshwork cells of the human eye as a model system for the study of cellular aging. Arch Gerontol Geriatr 9: 251-262, 1989.

25. Mandal AK and Pehere N: Early-onset glaucoma in Axenfeld-Rieger anomaly: Long-term surgical results and visual outcome. Eye (Lond) 30: 936-942, 2016. 